\title{
Protée
}

\section{Réexamen du parcours narratif : place et types de la sanction "Comment peut-on être Persan?"}

\section{Hamid Reza Shairi}

Volume 35, numéro 3, hiver 2007

Poétiques de l'archive

URI : https://id.erudit.org/iderudit/017482ar

DOI : https://doi.org/10.7202/017482ar

Aller au sommaire du numéro

\section{Éditeur(s)}

Département des arts et lettres - Université du Québec à Chicoutimi

ISSN

0300-3523 (imprimé)

1708-2307 (numérique)

Découvrir la revue

Citer cet article

Shairi, H. R. (2007). Réexamen du parcours narratif : place et types de la sanction : « Comment peut-on être Persan? ». Protée, 35(3), 89-101.

https://doi.org/10.7202/017482ar

\section{Résumé de l'article}

Par leurs diversités culturelles, les discours sont souvent le moyen de fournir à la théorie une force innovante. Le petit conte persan auquel nous nous intéressons ici nécessite une révision du régime narratif classique puisque le modèle standard de la narrativité semble insuffisant pour son examen. En effet, cette étude s'efforce de montrer comment le discours persan nous met en présence d'un parcours qui, nourri de la problématique de la tensivité et du corps, déplace les phases du schéma narratif en le faisant débuter par une sanction anticipée et manipulatoire. Nous verrons par la même occasion en quoi cette sanction procure à la narrativité une dimension sensible et phénoménologique. Cela nous permettra de constater que l'interaction des plans sémiotiques aboutit à l'insertion des dimensions axiologiques - impressive, émotionnelle et cognitive - dans le parcours narratif pour créer deux formes de vies distinctes : commencer par la fin ; finir par le commencement. 


\title{
RÉEXAMEN DU PARCOURS NARRATIF: PLACE ET TYPES DE LA SANCTION «COMMENT PEUT-ON ÊTRE PERSAN?»
}

\author{
HAMID REZA SHAIRI
}

Qui aurait pu croire que la sémiotique relèverait un jour le défi en tentant une réponse à la question posée il y a longtemps dans Lettres persanes de Montesquieu: «Comment peut-on être persan?» ([1721] 1992: 66). En vérité, il est impossible d'envisager une réponse définitive à une telle question, susceptible $d^{\prime}$ être renouvelée à travers toutes les époques. Le réexamen du parcours narratif et particulièrement de la place de la sanction, à partir des données culturelles de l'Orient, est pour nous l'occasion de montrer, d'une part, en quoi la sémiotique de la culture est capable de proposer de nouveaux parcours de sens et de souligner, de l'autre, à quelles «formes de vie» peut correspondre le fait $d^{\prime}$ «être persan». Ce sont en somme le principe $d^{\prime}$ « intertextualité » et les procédés de «transitivité intégrative» (Fontanille, 2006) - intégration des pratiques méta-sémiotiques ou interprétatives par des pratiques sémiotiques et confrontation des pratiques interprétatives à d'autres pratiques - qui rendent légitime une telle tentative.

Constituant l'un des instruments d'analyse les plus performants de la sémiotique en ce qui concerne la science du texte, le schéma de l'action est issu de l'analyse des contes populaires. Comme tout instrument d'analyse qui subit au cours du temps les effets de réexamen et de réadaptation à de nouvelles conditions de «vie», le schéma narratif, par souci de perfectionnement, est régulièrement confronté aux exigences de nouveaux régimes de construction du sens. Le modèle classique de la narrativité semble en effet insuffisant, inadapté quelquefois à la diversité des corpus provenant de cultures et de situations discursives très variées.

La sémiotique narrative classique a considéré le manque comme le déclencheur principal de l'action. D'où l'élaboration d'un parcours de programmation ou de manipulation qui cherche à réparer un tel manque et où la sanction se présente comme ultime étape. Cependant, le petit conte persan qui constitue I'objet de cette étude a bien montré que l'ordre du parcours narratif peut être renversé: ce qui devait constituer, par la logique des choses, I'aboutissement du procès narratif en constitue la teneur.

Cela nous met face à un déplacement des phases de la narrativité et change notre vision des choses et du monde. Ainsi placée au tout début du schéma narratif, la sanction manipulatoire fonctionne comme ce qui anticipe la fin par l'intrusion $d^{\prime}$ une force somatique. Ce rapprochement de la fin ne serait-il pas le moyen de nourrir le narratif par le tensif? C'est cela même qui modifie notre vision du monde, puisque le manque narratif standard semble être remplacé par l'excès (I'exercice anticipé de la force somatique) tensif; de même, I'oubli de l'activité tensive constitue un «accident» qui correspond cette fois au manque tensif, prive le parcours de sa force manipulatrice et participe à sa déprogrammation.

Dans l'ensemble, cette étude vise un triple objectif. Tout d'abord, il s'agira de voir en quoi le déplacement de la sanction à des fins manipulatoires garantit la compétence du sujet. Ensuite, il importera de montrer comment cette sanction manipulatoire inclut la dimension tensive et celle du corps propre dans le parcours narratif, lui offrant ainsi une dimension sensible et phénoménologique. Enfin, on évaluera dans quelle mesure la négligence de la sanction manipulatoire renverse le contenu narratif et nous met en présence d'un «accident» qui rend le parcours incertain, tout en ouvrant la voie à la dimension sociale, collective et imaginaire. 


\section{Bref rappel du parcours narratif classique}

Bien que les étapes du parcours narratif de Greimas aient vocation à l'universalité et qu'elles aient favorisé sur l'axe syntagmatique la mise en place d'un schéma narratif canonique, on ne peut s'abstenir d'évaluer la validité d'un tel système narratif pour des contes ou des énoncés appartenant à des ethnies ou cultures très variées.

Tous ceux qui s'intéressent à l'étude scientifique du récit et à la nationalisation de la question narrative ne peuvent s'empêcher de considérer les années 1960 comme marquant une vraie révolution narrative. De ce point de vue, cette période constitue une référence indéniable pour tout progrès méthodologique lié à la «narrativité 1 .

Comme nous le savons, V. Propp (1928) a été le premier à reconnaître pour tout récit, à partir d'un corpus de contes merveilleux russes d'Afanassiev, une organisation narrative sousjacente nommée «les trente et une fonctions du récit», dont les plus caractéristiques sont le manque et la liquidation du manque. Tout en s'inspirant des données théoriques de Propp, C. Bremond (1973) ouvre une nouvelle étape dans la genèse de la pensée sur la narrativité. Pour lui, les personnages occupent le premier plan et, étant donné qu'ils prennent sens par des rôles qu'ils occupent, la structure du récit ne peut plus reposer comme chez Propp sur la série d'actions et dépend fortement de «l'agencement de rôles ».

C'est en s'appuyant sur ces données que Greimas met en place sa structure narrative composée de trois "épreuves». "Épreuves», puisqu'il considère que le récit « est comparable au sens d'une vie » (cité dans Courtés, 2003 : 88). De cette façon, le changement et la transformation qui dépendent de I'action (l'épreuve décisive) résident au cœur de la sémiotique narrative de Greimas. D'ailleurs, chez Propp aussi l'action joue un rôle primordial. Cependant, ce qui distingue «l'action » greimassienne de celle de Propp, c'est le fait qu'elle a pour seule propriété le langage. Autrement dit, il s'agit pour Greimas de l'action «en papier ». C'est ce qui le mène, d'une part, à réduire les fonctions paradigmatique de Propp et, de l'autre, à consacrer son effort à la construction d'une syntaxe narrative qui, tout en reposant sur le concept d'actant, prend source dans la réalité langagière du discours et dépend de la dynamique narrative.

C'est ainsi qu'apparaît le schéma narratif de Greimas, qui, de portée générale, établit un ordre de parcours et une orientation des discours. D'abord structuré sous la forme de trois épreuves "qualifiante, décisive, glorifiante» (Greimas et Courtés, 1993: 131) -, ce schéma a été redéfini, donnant lieu à deux modèles narratifs encore plus fonctionnels: 1) Contrat, Compétence,
Performance, Sanction; 2) Manipulation ou Contrat, Action, Sanction. Si la priorité est donnée à la paire Sujet/Objet, c'est le plan de l'action (Compétence, Performance, Conséquence) qui révèle toute son importance. Et si le parcours Destinateur/ Destinataire, ou d'interaction entre les sujets, vient au premier plan, c'est la manipulation qui est mise de l'avant (Fontanille, 1998: 112).

Ainsi se dessinent trois grands domaines d'articulation sémiotique revendiquant l'autonomie des niveaux de parcours du sens par rapport aux données narratives. Il en résulte un élargissement du champ d'étude qui va au-delà du simple récit et qui peut concerner toutes sortes de discours.

Cependant, le point de convergence entre ces trois parcours est la place attribuée à la sanction. En effet, située au terme des schémas narratifs, cette dernière implique la présence d'un Destinateur. Soit ce dernier donne la récompense ou la punition à partir de la conformité ou la non-conformité de l'action réalisée par le sujet (sanction pragmatique); soit, sur un jeu de l'être et du paraître, il établit un jugement épistémique à propos de la vérité ou de la fausseté des faits accomplis (sanction cognitive); soit, enfin, il réagit positivement ou négativement à l'action menée en procurant un état d'âme euphorique ou dysphorique au sujet (sanction pathémique).

\section{La sanction comme déclencheur du parcours narratif}

Si nous voulons traiter la question posée au début de cet article, il importe de savoir si toutes les cultures et tous les discours partagent cette place accordée à la sanction et s'ils la traitent de la même façon. En vérité, nous sommes conscient du fait que rares sont les discours qui permettraient d'envisager la sanction comme le déclencheur principal de tout le parcours et de la situer à I'origine même de l'action. Mais un conte populaire persan, très connu depuis des siècles, intitulé Le Maître forgeron et son Apprenti et n'ayant qu'un statut oral, impose un sort différent au programme narratif et le fait commencer par une sorte de sanction pragmatique anticipée que nous pouvons considérer comme un moyen de manipulation ${ }^{2}$.

\section{Le Maître forgeron et son Apprenti}

Il était une fois dans un petit village, un Maître forgeron qui envoyait tous les jours, avant tout, son apprenti remplir la cruche à la fontaine. Chaque matin, avant le départ de celui-ci et avant de lui avoir remis la cruche, il lui donnait une gifle. Ensuite, I'apprenti partait vers la fontaine et revenait à la boutique avec la cruche remplie d'eau. Un jour, le Maître oublia de le gifler 
et l'apprenti, au moment du retour, fit tomber la cruche et la cassa. Dans l'entourage, ayant appris la nouvelle, les gens se dirent: "Sans avoir commis aucun tort, l'apprenti était condamnéà recevoir une gifle chaque matin; maintenant qu'il vient de casser la cruche, la mort sera certainement la moindre punition qui l'attend ». Mais, quand l'apprenti revint, le Maître ne fit aucun geste et ne se fâcha même pas. Les voisins, étonnés, en demandèrent la raison. Le Maître répondit: «Les autres jours, je le punissais avant son départ pour qu'il s'en souvienne sur tout le chemin et qu'il accomplisse bien sa tâche. Puisque j'ai oublié de le faire aujourd'hui, à quoi cela sert-il de lui en vouloir et d'avoir des regrets?». (Notre traduction) ${ }^{3}$

Ce conte semble bien commencer par une sanction pragmatique; or, cette sanction pragmatique anticipée (punir d'avance), que nous pouvons dénommer l'anté-sanction, constitue en fait une manipulation et même une forme de compétence. Autrement dit, nous avons affaire à une sorte de sanction, qui, au lieu d'être au service d'une évaluation finale, agit comme une figure de punition anticipée et manipule le sujet. À la sanction effective de la fin se substitue une sanction figurative du début. L'acte de frapper est en vérité une force figurative qui correspond à trois ordres discursifs. Premièrement, il apparaît comme une punition $d^{\prime}$ avance et constitue de ce point de vue une sanction pragmatique anticipée. Deuxièmement, il fonctionne comme ce qui met en branle le programme d'action et donne lieu de ce fait à une sanction manipulatoire. Troisièmement, il agit comme ce qui modalise l'action par rapport à un devoir-faire auquel le sujet ne peut plus échapper et met ainsi en place une sanction modalisante et préventive. Si nous mettons cette sanction en rapport avec l'évaluation qui se produit par les voisins quand l'action échoue, et qui est d'ordre imaginaire (imaginer la punition qui pourrait être appliquée par le Maître), nous nous trouvons en mesure de parler d'une post-sanction. $C^{\prime}$ est ce qui nous mène d'ailleurs à considérer l'acte de gifler, qui est préventif, comme une sanction inchoative et l'évaluation des voisins, qui est imaginaire, comme une sanction terminative. Comme nous pouvons le constater, nous sommes en présence d'une situation qui remet en question l'ordre canonique étant donné qu'elle déplace la sanction, multiplie ses fonctions en lui procurant une figure du début et une image de la fin, et l'enrichit sur tous les plans sémiotiques. À ce propos, deux remarques sont indispensables:

i) ce qui fait échouer ici le programme narratif, ce n'est ni le manque de l'action chez l'actant-sujet (il s'agit d'un parcours habituel auquel le sujet est familier étant donné qu'il se répète tous les matins), ni la présence quelconque d'un anti-Destinateur, d'un anti-sujet, ou d'un anti-programme (il s'agit du même parcours maintes fois réalisé sans aucun obstacle);

ii) une «erreur du Destinateur» explique cet échec puisqu'il oublie de donner la gifle et de mettre en œuvre la sanction préventive, ce qui démodalise le sujet, le désensibilise et finit par désémantiser l'acte. En tout cas, la programmation et la déprogrammation dépendent toutes deux de l'instance de la manipulation par sanction et de la présence ou de l'absence du corps du Destinateur. De ce point de vue, nous avons affaire à un conte qui ne propose qu'une seule quête (aller remplir la cruche à la fontaine) avec pour seul possible narratif une punition exercée par le Destinateur. En effet, une fois l'acte de gifler omis, le programme de quête sera suspendu et I'action échouera. Cela prouve que c'est une sanction manipulatoire qui ouvre la quête et la fait progresser, tandis que la négligence ou son absence la fait cesser, provoque à la fois la perturbation du programme d'usage (la cruche est cassée) et du programme de base (il n'y aura pas d'eau) et rend possible le passage à une sanction imaginaire.

Dans son étude sur la narrativité, en comparant les deux stratégies orientale et occidentale, E. Bertin attire notre attention sur le fait que l'Occident se caractérise par la logique du programme, un mode de pensée qui met l'accent sur l'événement et I'affrontement en tant qu'étape décisive de la situation narrative, alors que I'Orient et particulièrement la pensée chinoise sont reconnus pour leur obsession de la production de l'effet sur l'autre.

En épousant l'évolution du cours des choses pour en tirer parti, la stratégie chinoise s'inscrit dans une logique tensive et non narrative. Elle opère en effet sur le mode du déploiement continu qui sera porteur de transformation, mais déploiement non orienté à l'avance. La transformation opérée par le stratège s'étend ainsi dans la durée et c'est cette continuité qui lui donnera son effet. De ce fait, elle se caractérise en général par un tempo lent. On observe là un mode de devenir et de déroulement de l'activité stratégique qui s'oppose à notre tradition occidentale, fortement imprégnée du modèle narratif et de ses enchaînements d'étapes orientés vers un dénouement et ponctués de discontinuités. (Bertin, 2003 : 33)

Sans chercher à réfuter la thèse du sémioticien, nous voudrions avancer l'idée que, dans ce conte folklorique persan, la transformation ne dépend ni d'un programme conçu d'avance, ni de la durée ou de la lenteur. Tout au contraire, ce qui caractérise ici le procès et son résultat, c'est une non-durée et un tempo vif. 
Cependant, I'on ne peut pas nier la part de l'effet puisque l'application de la gifle peut être interprétée comme un «survenir», que J. Fontanille et C. Zilberberg définissent comme « coup » (1998: 118), ce qui correspond à l'anticipation de l'événement et à la représentation de la réalisation de l'action par le Destinateur avant même que le passage à l'acte n'ait lieu. On dirait que la sanction manipulatoire du début décide du sort de I'action; cela a pour résultat d'affaiblir la part de l'événement et $d^{\prime}$ accentuer le rôle de l'effet. Deux raisons justifient le fait de voir tout le procès sous l'emprise d'un «survenir»:

i) le déplacement de la sanction pragmatique vers le début du parcours pour la transformer en une punition anticipée, manipulatoire et tensive;

ii) I'intervention du Destinateur au commencement du procès que l'on peut définir comme une sanction préventive jouant le rôle à la fois de l'accélérateur et du régulateur par force de la trajectoire.

Ces deux procédures ont pour résultat de changer le statut du sujet-apprenti en un non-sujet. Selon E. Landowski, la première solution pour pouvoir manipuler l'autre en toute sécurité, c'est de le «ramener au statut d'un non-sujet» (2005: 24). Ainsi, il sera tout à fait juste de constater que le «survenir » oriente tout le parcours et le fait se dérouler dans le bon sens, non seulement parce qu'il crée un lien tensif entre l'actant-sujet et le parcours à accomplir, mais aussi du fait qu'il garantit la maintenance de la tensivité ${ }^{4}$ tout au long du procès ${ }^{5}$.

Le fait tensif relie ici le geste du Maître (l'intensité) et le déploiement spatial (I'extensité). En ce qui concerne l'activité somatique, nous sommes en présence du sensible qui est créé par l'atteinte au corps de l'autre. Par rapport à l'étendue spatiale, nous nous trouvons face à l'intelligible, c'est-à-dire à une évaluation qui concerne l'espace de l'autre et fait intervenir l'observateur social. À partir du rapport entre l'intensité (la gifle-tonique / l'absence de la gifle-atone) et l'extensité (la boutique-le proche / tout l'entourage social-le lointain), se produit une valence qui rend compte de la diffusion de la valeur. Du côté du rapport gifle/boutique (tonique/proche), nous avons affaire aux valeurs d'absolu, "éclatantes en intensité et concentrées en extensité» (Zilberberg, 2006: 27). Du côté du rapport absence de la gifle/ entourage social (atone/lointain), nous constatons des valeurs d'univers «médiocres en intensité et diffuses en extensité» (ibid.). À vrai dire, de même que l'intensité tonique met en branle le sujet, sémantise l'acte et met du sens dans le parcours somatique, de même l'intensité atone sémantise l'actant social, ouvre le champ interactionnel et donne lieu à une co-communication sociale (communication collective orientée par la même intention et au service d'un faire ensemble).

Dans tous les cas, les valeurs d'absolu garantissent le bon fonctionnement de I'action tout en empêchant l'emballement imaginaire de l'apprenti, tandis que les valeurs d'univers participent à l'incertitude du parcours de l'action et ouvrent la voie à l'emballement imaginaire de l'actant collectif. Ainsi, le rapport sensible/intelligible crée deux sortes de perspectives: la première est celle qui lie le sujet apprenti au Maître et aboutit à des valeurs limitatives (leçon pour un) - ce qui correspond à une perspective fermée -; la seconde s'explique par celle qui lie l'apprenti et le Maître à l'observateur social et débouche sur des valeurs illimitées (leçon pour tous) - ce qui correspond à une perspective ouverte.

Le tableau suivant présente le fait tensif que nous venons de décrire:

Schéma de la structure tensive et de la mise en perspective

\begin{tabular}{|c|c|c|c|c|}
\hline Niveaux & $\begin{array}{c}\text { sensible } \\
\downarrow \\
\text { intensité }\end{array}$ & $\begin{array}{c}\text { intelligible } \rightarrow \\
\downarrow \\
\text { extensité } \rightarrow\end{array}$ & valence & perspective \\
\hline Sous-niveaux & $\begin{array}{c}\text { tonicité } \\
\downarrow \\
\text { tonique } \\
v s \\
\text { atone }\end{array}$ & $\begin{array}{c}\text { déploiement spatial } \\
\downarrow \\
\text { proche } \rightarrow \\
v s \\
\text { lointain } \rightarrow\end{array}$ & $\begin{array}{c}\text { valeurs } \\
\downarrow \\
\text { valeurs } \mathrm{d}^{\prime} \text { absolu } \\
v s \\
\text { valeurs } \mathrm{d}^{\prime} \text { univers }\end{array}$ & $\begin{array}{l}\text { un } v s \text { tous } \\
\text { fermée } \\
\text { ouverte }\end{array}$ \\
\hline
\end{tabular}

En effet, l'application de la force (la gifle) au corps du sujet modifie son statut, affecte son parcours et fait de celui-ci un sujet sensible tout en créant une différence de potentiel. Aussi, cette force change la sensori-motricité d'un tel sujet car elle lui procure une certaine compétence, qui non seulement détermine le dynamisme de l'action, mais aussi l'oriente dans sa seule visée (apporter la cruche remplie d'eau à la boutique). En tout état de cause, nous avons affaire à une stratégie qui fait prévaloir l'effet sur l'événement par la production du choc et du vif. C'est pourquoi tout obéit à une logique tensive. Le parcours de l'apprenti ne subit la défaite que lorsque la sanction manipulatoire du début n'est pas respectée. Le déséquilibre provient alors du manque du «survenir». Il faut que l'éclat du début se change en matité pour que nous soyons confrontés à la détérioration du procès et à l'arrêt de l'événement. Tout se passe comme si l'intensité était le seul garant de la trajectoire narrative et que son absence impliquait la déprogrammation. Une distinction demeure à I'origine de la désorientation du parcours: tant que le «survenir » a lieu et que l'éclat fonctionne comme une valence ${ }^{6}$ tensive prenant en 
charge le devenir du procès, nous avons affaire à un parcours identique sans qu'aucune résistance ne vienne perturber la situation narrative; mais, dès que l' « identique » est remplacé par le «distinct», le cours du devenir s'arrête et nous sommes conduits à l'échec. Autrement dit, le dysfonctionnement de la force tensive, que l'on peut identifier à l'absence de la sanction manipulatoire et au relâchement de l'éclat, entraîne la perte du contrôle de la situation narrative et fait échouer l'action. Cela montre bien que la punition placée au commencement du programme correspond à une visée tensive responsable de la régulation de tout le devenir en cours et de la manipulation du sujet de l'action.

En ce qui concerne la temporalité, la totalité du conte semble être régie par deux axes différents: i) «tous les jours»; ii) «un jour». Le premier est celui de la successivité, de la continuité et de l'identique (le même). Le deuxième se caractérise par la singularité, la simultanéité, la discontinuité et le non identique. Cette temporalisation témoigne de deux schémas narratifs distincts sur le plan paradigmatique et récurrents sur le plan syntagmatique. Il s'agit en fait, dans les deux cas, du même parcours opposable au plan paradigmatique. À la sanction manipulatoire s'oppose I'absence de sanction et, à la saisie axiologique (la conjonction avec l'objet de valeur - la cruche remplie d'eau), s'opposent, d'une part, la saisie impressive («se livrer à l'impression immédiate »[Fontanille, 1999: 227]) des voisins («la mort... l'attend») et, de l'autre, la saisie cognitive du Maître (le raisonnement qui fait savoir que tout regret concernant la perte de l'objet est inutile). Les schémas suivants résument l'ensemble de ce que l'on vient de constater sur le rôle et la place de la sanction:

Schémas des parcours de la sanction

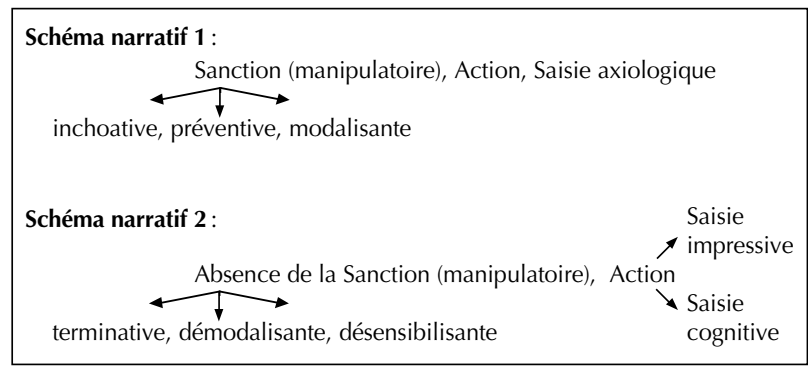

Cependant, on ne peut ignorer le fait que la temporalité successive est marquée par une forte tensivité, étant donné qu'à son origine réside une sensori-motricité somatique de l'ordre du «survenir » et qu'elle est déterminée par l'itérativité. En revanche, la temporalité simultanée est identifiée au relâchement et à I'absence de tensivité. Cela indique que la présence appartient ici au domaine du continu et que l'absence s'inscrit dans le champ du discontinu. Cela montre également que la présence relève de I'intensité et produit des effets toniques, alors que l'absence est de l'ordre de l'extensité et donne lieu à des effets atones. À ce sujet, $\mathrm{H}$. Parret ne manque pas de préciser que

[...] la valeur énonciative de présence est imposante. La reconnaissance ou le saisissement d'une "présence » est une affaire hautement affective, voire [érotique]. Les présences sont pathémisées et jamais les corrélats d'états purement cognitifs. (2001: 10)

On ne peut pas évoquer ici la question de la temporalisation sans établir le lien avec la procédure spatiale. «Est présent ce qui est là, hic et nunc, in vivo» (ibid.: 61). En réalité, l'espace intervient de deux manières dans les schémas narratifs du Maître forgeron et son Apprenti: dans un premier temps, en tant qu'espace familier et circulaire qui va de la boutique à la boutique, il participe au bon déroulement de l'action et s'identifie à l'espace intime de la réalisation; dans un deuxième temps, en tant qu'espace étranger et distinct, il marque la rupture de l'événement et est semblable de ce point de vue à l'espace social de la virtualisation. On pourrait maintenant assimiler ces deux types d'espace à deux sortes de visées qui se dessinent à partir de chacun des parcours narratifs mentionnés: à l'espace de la réalisation correspond une visée tonique; à l'espace de la virtualisation, une visée atone. Pour dire comme Fontanille et Zilberberg (1998: 93), le premier coïncide avec «le proche» et constitue une spatialité immédiate, et le deuxième est en concomitance avec «le lointain » et nous met en face d'une spatialité distante.

Dans les deux cas de figure, qu'il s'agisse d'une tensivité forte (présence) ou d'une tensivité faible (absence), nous sommes confrontés à une quête régie par la modalité du /vouloir/ ${ }^{7}$. Il est à noter que ce /vouloir/ apparaît sous forme d'un /faire-vouloir/ étant donné qu'il est infligé par le Destinateur à l'actant-sujet. Mais il est aussi évident qu'un tel /vouloir/ se manifeste sous deux conditions: i) par la transmission du programme à l'actant (le fait de lui remettre la cruche chaque matin); ii) par la sanction manipulatoire (I'application de la gifle) et la transmission de la dynamique. Ainsi, l'application modale du vouloir trouve deux sources différentes dans notre conte: I'une perceptivo-somatique (la gifle qui sert de moteur au mouvement et qui détermine le parcours du sujet) et l'autre éthico-cognitive (la cruche qu'il faut remplir est le lieu d'inscription d'une trajectoire et d'une loi qui considère l'eau comme le signe de la vie pour la boutique «tous les jours, avant tout...»). Selon les termes de P. A. Brandt, ce Destinateur serait «investi comme petenta (une loi ou une 
divinité) » (1992 : 89). D'ailleurs, si l'on assimile le Maître forgeron à une divinité, on serait amené par la suite à interpréter le vouloir accélérateur (la gifle) comme un pouvoir qui garantit la compétence de l'apprenti. Ainsi, I'on ne peut plus nier la présence modale du pouvoir-faire que le Destinateur est en mesure de transmettre au sujet par l'exercice de la force somatique. De toute façon, dans le cas de la temporalité continue dotée de la vision tonique, les deux aspects du vouloir sont présents. C'est ce qui fait apparaître le Destinateur dans le rôle d'un sujet opérateur. Cela crée une dynamique très compliquée dont Brandt propose la définition suivante:

1) elle [cette dynamique] est capable de vouloir pour une autre, au nom d'une autre, de transférer ses pulsions (de vie ou de mort) sur une autre dynamique, donc d'échanger de larges séquences de sa mémoire modalisée contre celle d'une autre dynamique - par une sorte de mimétisme; [...] son vouloir est transitif, transmissible, actif comme un faire-vouloir ; [... 2) elle dispose [...] d'un espace de communication et d'influence qui fonctionne comme un fragment d'espace de contrôle, où son image intentionnelle d'un espace-temps voulu-pour-l'autre affecte les variables externes jusqu'à les amener à la reproduire sous la forme d'une contrainte fatale, d'un chemin objectif, passant exactement là où se trouve l'autre en l'honneur de qui l'image intentionnelle était formée. (Ibid. : 102-103)

Mais, dans le cas de la temporalité discontinue, privée de l'intensité, seule la source éthico-cognitive du vouloir est impliquée. Cela nous amène à constater qu'il est seulement question, en ce qui concerne la visée atone, d'une pré-modalisation (métavouloir) puisqu'il y manque la force nécessaire pour changer la dynamique du sujet et le rendre compétent (l'absence de la dimension perceptivo-somatique et donc du pouvoir-faire). C'est pourquoi la quête qui nous préoccupe ici relève d'une même instance sémiotique dédoublée: celle d'une présence étirée entre le réel et le virtuel. Le tableau ci-dessous montre que les deux types de temporalité à l'œuvre dans notre conte correspondent chacun à divers effets de sens qui dépendent eux-mêmes de différents niveaux sémiotiques.

Comme nous pouvons le voir, la sanction manipulatoire du début de parcours non seulement régule le processus en le surveillant avec soin, mais en plus l'affecte sur de nombreux plans sémiotiques qui méritent une réflexion plus approfondie.

\section{Une narrativité tensive}

Sur le plan narratif, nous sommes en présence d'une quête instaurée par un Destinateur initial. Ce dernier apparaît comme une divinité qui décide du sort du Destinataire-sujet ainsi que de I'ensemble du parcours par le biais de la punition anticipée. L'exercice de la sanction manipulatoire, placée tout au début du procès, change notre conception de la narrativité pour laquelle la sanction trouvait toujours sa place à la fin du parcours narratif. Ce changement modifie l'image que l'on se faisait du sujet de la quête en le considérant comme "partenaire du dialogue et d'échange» (Geninasca, 1997: 43). En vérité, notre sujet occupe ici le statut $d^{\prime}$ une machine qui ne peut fonctionner que selon le programme mis à sa disposition. Pour un tel sujet, la question de la crédibilité, comme le suppose J. Geninasca, ne se pose pas.

Devenir crédible c'est, pour le sujet du faire persuasif, obtenir la reconnaissance de son identité, de la permanence dans le temps du vouloir qui le lie, indépendamment des circonstances, aux valeurs qu'il assume et qu'il suppose assumées par le sujet judicateur à I'arrêt duquel il se soumet. (Ibid.)

La punition du début efface l'identité du sujet parce qu'il ne restera rien à évaluer. La transformation ne dépend ni de la compétence ni de la performance du sujet. Tout au contraire, elle repose entièrement sur l'activité somatique du Destinateur initial. Ce qui est

Présentation de la temporalité et des niveaux sémiotiques du conte

\begin{tabular}{|c|c|c|}
\hline temporalité & tous les jours & un jour \\
\hline niveau narratif & $\begin{array}{c}\text { sanction manipulatoire } \rightarrow \text { action } \\
\rightarrow \text { saisie axiologique }\end{array}$ & $\begin{array}{c}\text { absence de la sanction manipulatoire } \rightarrow \text { action } \\
\rightarrow \text { saisie impressive et cognitive }\end{array}$ \\
\hline niveau modal & $\begin{array}{c}\text { faire-vouloir } \\
\text { devoir-faire } \\
\text { pouvoir-faire } \\
\text { néta-vouloir } \\
\text { ne pas pouvoir-faire }\end{array}$ \\
\hline niveau tensif & visée tonique & visée atone \\
\hline niveau spatial & proche & lointain \\
\hline niveau de présence & réel & virtuel \\
\hline
\end{tabular}


à l'origine de la conformité du sujet à l'univers de la réalisation ne tient pas à l'action pour laquelle il est programmé. C'est l'acte tensif du Destinateur qui s'avère décisif pour tout le parcours. De ce point de vue, la signification peut être considérée non plus comme quelque chose à venir, mais comme une donnée que le sujet judicateur impose dès le départ, avant même que le sujet ne soit plongé dans l'univers de l'action.

Cependant, le conte que nous étudions met en place trois types de Destinateurs.

1) Un Destinateur initial (individuel) qui communique au sujet un programme à accomplir dont la Vérité est garantie par I'application de la force tensive. En effet, ce Destinateur fait en sorte que la séquence contractuelle et la sanction existent au même moment. Tout se passe comme si le contrat était signé et mis en œuvre par la sanction. Cette confusion des séquences ne peut répondre qu'à une seule exigence sémiotique: recourir à la tensivité forte afin de garantir le bon déroulement du procès narratif. Évidemment, cette tensivité s'obtient ici par la superposition d'une séquence (contractuelle) à une autre (sanction).

2) Un Destinateur intermédiaire (social) qui assume le faire interprétatif quand la cruche est cassée. Il n'a pas le même statut sémiotique que le premier. II signifie par une saisie impressive, son faire est d'ordre cognitif et il prend source dans le faire pragmatique du Destinateur initial ("Chaque matin, [...] il lui donnait une gifle»). Il témoigne de l'intention de communication qui s'établit, d'une part, entre les gens du village (co-communication) et, de l'autre, entre ceux-ci et le Destinateur initial (intercommunication: «Les voisins, étonnés, en demandèrent la raison »). De plus, il s'inscrit à l'intérieur de la fonction jugement puisqu'il débouche sur un faire interprétatif voyant la mort comme la seule punition possible pour le Destinataire-sujet («maintenant qu'il vient de casser la cruche, la mort sera certainement la moindre punition qui I'attend»). On peut interpréter la présence de ce Destinateur intermédiaire comme la mise en relief, une fois de plus, de la séquence sanction sur laquelle repose toute la dimension narrative. Le jugement médiateur, fondé lui-même sur la punition anticipée, débouche sur une justification du Maître forgeron. Nous sommes donc ici en présence de deux activités d'ordre cognitif appartenant à deux registres différents: le faire pragmatique et le faire observateur. De fait, le médiateur apparaît dans le rôle d'un actant observateur et collectif. «Un actant collectif se définit pour sa part comme une collection d'acteurs individuels dotés d'une compétence modale ou d'un faire commun» (Alonso Aldama, 2000: 51). Notre actant collectif se trouve caractérisé par le trait modal de croire qui doit son existence à la manifestation figurative d'une donnée aspectuelle définissable comme une sanction itérative (la répétition de la gifle). On peut ainsi mentionner la débauche d'une séquence narrative qui débute par la sanction et aboutit à une saisie cognitive. Cette dernière prend la forme d'une suite syntagmatique qui correspond à un ordre de faire: observateur, interprétatif, évaluatif. L'observation n'a de sens que par rapport à l'activité tensive du sujet opérateur. Elle a ainsi pour fondement une vérité factuelle qui est à l'origine de l'attente de l'actant collectif et qui oriente son savoir autant qu'il aboutit à un croire: "la mort sera certainement la moindre punition qui l'attend».

Nous constatons que le savoir et le croire déterminent le faire interprétatif du Destinateur-observateur. Situé entre le Destinateur initial et le Destinateur final, le Destinateur intermédiaire est cette figure collective qui, allant, en ce qui concerne l'action du sujet, du mode existentiel du réel à celui du virtuel, se place dans l'ordre du continuum tensif ${ }^{8}$. En effet, I'inscription du Destinateur collectif dans la dimension tensive se manifeste par son faire interprétatif que l'on peut assimiler à la supposition du plus haut degré de l'acte tensif: infliger la mort. Selon ce type d'actant, la mort serait l'aboutissement logique d'un parcours tensif qui commence par la gifle.

3) Mais on peut supposer aussi la présence d'un Destinateur final qui introduit une rupture dans cet ordre tensif et fait apparaître la discontinuité dans le continu; ce qui s'explique d'ailleurs comme une frustration à l'attente du sujet.

Ce qui arrête le cours de la tension et s'oppose au continu tensif imaginé par l'actant collectif relève, nous semble-t-il, d'une différence de conception du statut de la valeur. Pour le Maître forgeron, la tensivité ne peut être qu'au service d'une valeur dont la vérité est à garantir. Autrement dit, la force tensive n'est qu'un soutien pour des valeurs à venir. Les valeurs dont la vérité ne peut plus être sauvée ne méritent aucune tensivité et, par voie de définition, aucune sanction pragmatique («à quoi cela sert-il de lui en vouloir et d'avoir des regrets»). Avec l'écroulement de la valeur s'effondre également toute tâche tensive et se ferme logiquement la voie à la passion (pas de regrets). II n'y a pas d'effet passionnel, d'une part, parce qu'il n'y a pas de valeur en perspective et, de l'autre, parce que nous sommes dans un univers sémiotique qui ne donne lieu à aucune polarisation thymique d'ordre euphorique ou dysphorique. "L'effet passionnel résulte [...] de 
la conjugaison de ces deux propriétés, I'affect et la valeur» (Fontanille, 1998: 202).

Ainsi, du point de vue affectif, trois moments sont à distinguer dans le conte:

1) Tout d'abord, on remarque un temps initial qui fait apparaître un Destinateur scindé en deux étant donné qu'il se caractérise par deux formes de vie: I'une embrayée et l'autre débrayée. L'embrayage fonctionne comme un rappel tensif et fait revivre le Destinateur (tous les jours) dans le plan affectif, ce qui se rapporte à la scène passion-action-valeur. Le débrayage correspond au relâchement tensif et donc à l'absence du plan affectif, ce qui nous confronte à la situation non-passion-actionnon-valeur.

2) Ensuite, on observe un temps médiateur entendu comme la présence d'un Destinateur collectif qui ne voit qu'une continuité dans le parcours tensif. Cela signifie imaginer une scène complètement dysphorique: passion-action-passion (gifle, nonvaleur, mort). Pour être plus précis, on peut souligner que l'absence de la gifle peut être considérée comme une alternative qui ouvre la voie à une esthésie puisqu'elle nourrit dès le départ le parcours d'émotion en renversant l'axiologie. Cette absence supprime la compétence du sujet et dote le parcours d'une alternative émotionnelle en rendant les voisins capables d'imaginer une sanction terminative.

3) Enfin, on constate un temps final qui fait réapparaître le Destinateur initial, mais cette fois avec la décharge affective une manière d'établir la phorie (pas de regrets) et d'abandonner ainsi la dimension affective au profit de la dimension cognitive. Cela peut s'interpréter comme la présence de l'ordre scénique passion-action-cognition. Nous avons ainsi affaire à une circulation des scènes: la scène du Maître nourrie d'une force tensive (tonique/atone); celle de la collectivité caractérisée par l'émotion ou l'esthésie; celle, enfin et à nouveau, du Maître par une voie phorique où se dessine la saisie cognitive.

\section{L'instance du corps}

Puisque tout commence par une prescription tensive d'ordre gestuel (la gifle), on peut dire que le plan somatique déploie toute son ampleur dans l'énoncé. En effet, la punition liminaire, d'une part, déclenche le programme narratif tout en garantissant son bon déroulement jusqu'à la réalisation et, de l'autre, sert à la transmission du savoir à l'actant-sujet. C'est ce qui impose d'ailleurs une lecture phénoménologique du texte. Tout se passe comme si le phénoménal devenait le lieu approprié où s'inscrit le plan de l'action et comme s'il fonctionnait en tant que centre déictique autour duquel s'organise tout le parcours narratif depuis la sanction jusqu'à la fin. C'est pourquoi, une fois le corps absent, nous serons confrontés à la déprogrammation et l'action ne pourra pas aboutir. À ce sujet, J.-C. Coquet affirme que «I'instance de base, $\mathrm{c}^{\prime}$ est le corps [...]. C'est [...] en fonction de sa position, de ses actes que le sens prend forme.» (1997: 114). Ainsi, le corps du Destinateur se situe à l'origine de tout le parcours, mais on dirait qu'il délègue par l'acte somatique son pouvoir et son savoir au sujet. De fait, dans le rapport corps à corps qui s'établit entre les actants, le Destinateur impose toutes ses tensions au sujet, qui, en les mémorisant, sera capable de mener le parcours à sa fin. Autrement dit, il transforme le corps du sujet en un lieu possible du parcours et de l'action. II en résulte que le fonctionnement du corps est dédoublé : nous avons affaire, d'une part, à un corps source et cible qui s'énonce comme phénoménal et, de l'autre, à celui qui mène le parcours narratif et qui s'avère sémiotique. De toute façon, il est évident qu'une seule instance régit les deux: le corps du Destinateur. II ne faut pas considérer ce corps propre du Destinateur, qui prend sens dans son acte tensif et qui impose ses règles au monde, comme privé de la «capacité réflexive » et donc de la force assertive. En fait, la sanction manipulatoire placée et répétée au début du parcours remplace l'assertion prédicative et ne laisse aucun lieu de doute à la présence d'un ego qui s'affirme par son intervention somatique et tensive sans pourtant se dire ego par l'activité de la prédication. Comme le précise F. de Chalonge: "Dans ce qui est ainsi conçu comme le domaine de "I'anté-assertif", le corps prend une place centrale» (1996: 105). Ainsi, en tant qu'instance fondatrice de la narrativité, la sanction anticipée est ici responsable du déchirement corporel et de la diversité de I'identité ${ }^{9}$ actantielle. Elle distingue deux corps qui interviennent à deux niveaux différents. Un corps source (le Destinateur) que J. Fontanille identifierait au «Moi-chair» :

Le Moi-chair étant l'instance de référence, l'identité postulée mais toujours susceptible de se déplacer, il adopte une ou plusieurs positions, et il est le siège et la source de la sensori-motricité qui détermine toute visée et toute saisie sémiotiques. (2004: 37)

Et un corps cible que nous reconnaissons comme le Moi-corps (le sujet de l'action) puisqu'il représente une figure qui prend sens à la fois dans le Moi-chair qui le guide et par l'acte qu'il est contraint de mener. Le premier correspond au corps référent qui détermine l'énergie nécessaire au parcours et à son orientation aussi bien que l'inertie qui finit par y nuire. Le deuxième est assimilable au corps référé qui mène l'action sous le contrôle du 
premier et s'abandonne à la défaite quand la tension manque. Autrement dit, le modèle de la production de l'acte reposera ici, du côté du Destinateur, sur l'interaction entre le Moi-chair et le soi-idem (une intensité maximale - la gifle - et une extensité minimale - le proche), d'une part, et entre le Moi-chair et le soiipse (une intensité minimale - l'absence de la gifle - et une extensité maximale - le lointain), de l'autre. Il dépend également, du côté du sujet, de l'interaction entre le Moi-corps et le soiidem (la réception de la gifle et la continuité dans le parcours), $d^{\prime}$ une part, et entre le Moi-corps et le soi-ipse (l'absence de la gifle et l'arrêt du parcours), de l'autre. Ces interactions auront pour résultat la mise en place de deux types de valences: une valence d'intensité et une valence d'étendue. La corrélation entre ces valences peut donner lieu à des valeurs différentes. Du côté de la tensivité forte (pratique et réception de la punition) en corrélation avec l'étendue minimale (le proche et le parcours sans faute du sujet), nous sommes en présence des valeurs d'absolu que l'on peut définir comme des valeurs de concentration, d'effort, de vigilance, de maintenance et de succès. Du côté de la tensivité faible (I'absence et la non-réception de la punition) en corrélation avec l'étendue maximale (le lointain et l'arrêt de l'action), nous nous trouvons face à des valeurs d'univers que l'on peut assimiler à des valeurs de déconcentration, de distraction, de déviance, de défaillance, de défaite et de changement. Le schéma suivant indique bien les deux types de valeurs que la corrélation tensive peut mettre en jeu:

Schéma tensif de mouvements d'ascendance et de décadence

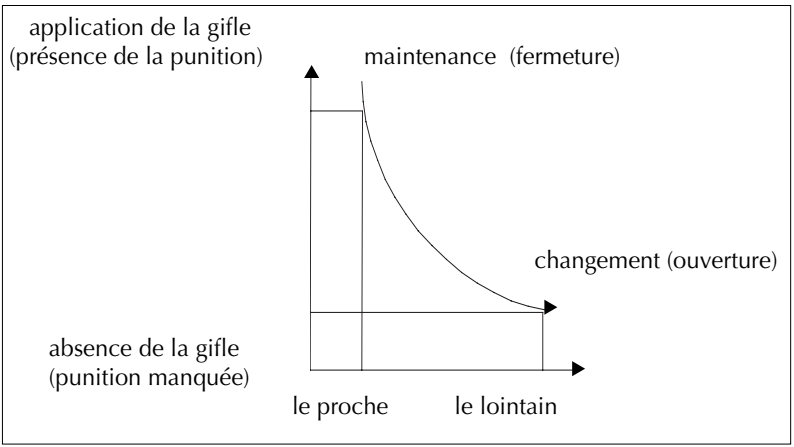

Comme le précise J. Fontanille, quand il y a, à partir de l'implication de la force tensive par le Moi-chair, dans le parcours de l'actant-sujet, "le recouvrement de chaque nouvelle phase sur la précédente» (ibid.), nous accédons à un type de perspective qui se qualifie comme la saisie et crée «l'identité des rôles». Et, dès que la sanction anticipée est interrompue et que nous sommes confrontés à la non-procuration de la force tensive, la procédure narrative s'arrête et avec cette rupture se créent un changement et un nouveau programme qui s'ouvrent sur l'imaginaire collectif. C'est ce qui lie d'ailleurs l'actant social à la perspective d'une nouvelle sanction : la mort. Cet oubli de la pratique de la punition que l'on peut assimiler à l'inertie constitue un manque pour l'actant sujet, non pas sur le plan narratif mais sur le plan tensif. Il est évident qu'une telle lacune cause la perte de tous les repères narratifs chez l'actant sujet puisque la position référentielle du Moi-chair en tant que source de l'énergie est absente. Cela aboutit au trouble de l'identité chez le sujet étant donné que les trois types d'identités susceptibles de décrire «le devenir de l'actant » - la référence tensive, la saisie et la visée sont absents.

Dans l'ensemble, à la sanction anticipée (la prise de position et l'instance de référence) et à son absence correspondent deux zones différentes de la narrativité.

1) La zone efficacité où le Moi-chair fonctionne comme le vrai et seul responsable de l'action de l'actant apprenti - sa programmation, son acheminement et sa réalisation - par le recours à l'activité tensive de la sanction. Ainsi, il se rallie au sujet par une implication somatique et domine tout son parcours. En effet, le Moi-corps prend sens dans cette transition tensive et construit à l'intérieur de la zone Moi-chair celle de soi-idem. Cela aboutit à l'installation d'une identité qui se définit par répétition et $d^{\prime} u n$ actant qui «sait sa leçon» et la répète sans faute. De cette façon, s'établit une zone de conformité où l'actant applique parfaitement les «scripts ». Selon J. Fontanille, «cette zone où la mémoire sémiotique de l'actant est entièrement consacrée à sa programmation est aussi celle de l'efficacité et de l'économie narrative» (ibid.: 41). Nous sommes dans l'ensemble dans une zone de progression et de réalisation due à la tensivité somatique.

2) La zone inefficacité où le Moi-chair, par absence d'activité tensive, cause la déprogrammation et l'échec du parcours de I'actant apprenti. C'est dans cette zone que l'actant connaît des troubles d'identité et n'est plus apte à mener l'action. On dirait que l'absence de la sanction anticipée empoisonne toute la zone de la narrativité, la rend non conforme, aboutit au dysfonctionnement de la mémoire sémiotique de l'actant et éteint son parcours. Cela non seulement prive l'actant de toute chance de saisie ou de visée, mais se situe en outre à l'origine de l'inefficacité narrative. Nous sommes dans une zone de dégradation et d'échec qui tient à l'absence de la tensivité somatique. 


\section{La tensivité aspectuelle}

La manière dont le conte met ici son objet en perspective paraît très curieuse. Tout d'abord, il oppose, par rapport à la sanction anticipée, deux temporalités dont la première («tous les jours») appartient à la dimension aspectuelle de l'itérativité et la deuxième ( «un jour»), à celle de la ponctualité. L'aspect itératif est caractérisé par une intensité maximale et sert à recharger le procès. Il apparaît, en fait, comme un écoulement continu du temps qui vient rythmer et équilibrer le programme en jeu, tandis que l'aspect ponctuel est définissable par une intensité minimale dont le rôle est de décharger le procès. La ponctualité marque une rupture dans l'écoulement du temps et contrebalance de surcroît les pulsions rythmées et régulatrices de l'action jusqu'à son arrêt total.

Ensuite, par rapport au procès lui-même, la temporalité itérative correspond au sème aspectuel de la perfectivité, alors que la temporalité ponctuelle correspond à celui de l'imperfectivité. D'une part, une force centripète et conductrice se dessine, lie le sujet au point de référence et mène le programme vers son achèvement (aspect terminatif). D'autre part, une force centrifuge s'annonce (aspect inchoatif), détache le sujet du centre de référence et arrête le cours du programme. Le couple itératif/perfectif donnerait lieu au massif puisque nous sommes en présence $d^{\prime}$ une accumulation de procès identiques et terminatifs. Le couple ponctuel/imperfectif, quant à lui, actualiserait le rare et l'épars car nous nous trouvons face à un procès dévié, dispersé et qui a échoué. En effet, le perfectif est lié à la réalisation de l'action et, de ce point de vue, il serait affaire de visée, alors que l'imperfectif nous ramène au virtuel et nous situe en ce sens du côté de la saisie.

\section{Zilberberg attire notre attention sur le fait que}

[...] le perfectif est une manifestante aspectuelle qui a pour manifestée un tempo rapide, et à son tour ce tempo rapide signifie que l'énonciateur fait savoir à l'énonciataire qu'il n'a pas de temps à donner, c'est-à-dire à "perdre», qu'il ne s'étendra pas, ne s'attardera pas sur... et inversement : I'imperfectif signifie par le choix de la lenteur cette fois que le sujet s'ouvre à la durée, que l'énonciateur comme l'énonciataire vont prendre leur temps, tout leur temps.

(1991: 102)

En accordant cette remarque à l'objet de notre étude, nous constatons que la gifle, en tant que détermination du procès, nous fait accéder au perfectif puisqu'elle anticipe le résultat de l'action, garantit son dynamisme, empêche son égarement et la rend mécanique, car tout se déroule comme il se doit. Toutes ces indi- cations vont dans le sens de l'accélération du procès qui s'affirme «indivisible». Tout se passe comme si l'action dépendait non plus du temps de l'action, mais de l'aspect perfectif, qui prend sens lui-même dans un tempo rapide défini par le coup (la gifle).

Tout au contraire, I'absence de la gifle espace le temps du procès, donne à l'action la possibilité d'être «divisible», rend le Destinataire-sujet distrait, impose au parcours narratif une certaine lenteur, qui ne peut que coïncider avec l'imperfectif, et finit par s'ouvrir sur une scène émotionnelle collective. On dirait que la tensivité aspectuelle enlève au temps linguistique son effet de durativité («tous les jours» ne produit plus la valeur temporelle qu'on lui reconnaît). De même, on dirait que l'absence de cette tensivité surajoute à la valeur du temps linguistique ( « un jour»), annule son effet de brièveté (par rapport à tous les jours) et impose une temporalité étendue qui va saisir (comparaison des deux temps par les voisins) toutes les cases du procès: les procès précédents menés avec succès et le procès échoué. Cela montre bien que l'imperfectif relève de l'instabilité et nourrit l'imaginaire étant donné qu'il n'est pas orienté, faute de la tensivité aspectuelle. Ainsi, il est facile de remarquer que cette dernière a la capacité de déjouer l'ordre temporel et finit par conférer au discours non pas un temps qui lui serait propre, mais un tempo qui lui est propre. En outre, reconnaître que l'emprise du Destinateur sur le procès le stabilise et équivaut au perfectif, signifie que nous avons affaire à un parcours totalement embrayé. C'est ce qui prouve d'ailleurs qu'un tel procès ne peut pas être subjectif, même si la part du sujet dans l'aboutissement de l'action est considérable. L'embrayage est aussi ici d'ordre tensif puisqu'il est réalisé par I'interaction corporelle de la gifle. II garde en fait le Destinatairesujet en liaison constante avec l'ici-maintenant créé par l'application de la force tensive. C'est pourquoi le temps se transforme en tempo pour ne viser que le résultat de l'action. On peut donc en conclure que la sanction anticipée a pour fonction, dans le texte étudié, de rendre le procès en question embrayé, perfectif, objectif, stable, indivisible et réalisable.

Mais une fois le parcours narratif dégagé de l'emprise du Destinateur, se crée un nouveau temps subjectif indépendant du «maintenant» tensif de l'action dictée par le Destinateur. C'est ce qui nous fait passer de l'embrayage au débrayage. En effet, I'absence de la sanction manipulatoire change la notion de temps, la libère de ses contraintes narratives, fait intervenir l'imaginaire collectif, rend le sujet responsable de ses actes et lui confère un nouveau statut qui est celui du sujet autonome. Il en résulte que I'absence de la sanction manipulatoire permet de rendre le procès débrayé, imperfectif, subjectif, instable, divisible et virtuel ${ }^{10}$. On 
voit bien comment la tensivité aspectuelle qui provient de la sanction anticipée permet d'outrepasser la notion de temps et de I'inscrire dans l'ordre du tempo. C'est exactement sous l'effet de cette même tensivité que l'ordre temporel est inversé: 1) ce qui a été évalué, sur le plan linguistique, comme une temporalité longue est assimilable, sur le plan sémantique, à une temporalité brève, vu l'aspect mécanique du procès; 2 ) ce qui a été considéré linguistiquement comme une temporalité brève cède sémantiquement sa place à une temporalité longue, étant donné l'indépendance du procès et I'autonomie du sujet. Ici, tout se passe comme si le sens de la vie dépendait non plus de l'ordre, de la régularité et de la continuité, mais de l'oubli provoquant le discontinu, l'absence, le désordre, l'imaginaire et l'inattendu. En effet, en tant que «contingences de la vie pratique» et ayant un impact sur le bon ou le mauvais déroulement des procès, l' «oubli » (quoique considéré comme involontaire) devrait être pensé comme l'un des principes d'articulation sémiotique, avec cette différence qu'au lieu d'appartenir à des stratégies de programmation et à des parcours de sécurité, il relève de l'imprévu et de I'«accident».

C'est pourquoi l'absence de la sanction anticipée pourrait être interprétée comme une échappatoire à la routine et à la pratique d'une vie trop soumise au rythme régulier de la programmation, ou encore comme un souffle de vie et une fuite devant le régime contraignant d'une itérativité insensée. Le sens provient donc, dans notre cas, non pas de la présence, mais de l'absence et de ce que E. Landowski nomme le «risque».

Au même titre que la régularité, principe présupposé par toute forme programmatique de l'interaction, que l'intentionnalité, base nécessaire pour toute manipulation stratégique, et que la sensibilité, condition de toute interaction en forme d'ajustement, l'aléa constitue de la sorte le principe fondateur d'un régime de sens et d'interaction autonome - le quatrième (et dernier) - à placer sur le même plan que trois autres: le régime de l'accident. Curieusement, nous le connaissons depuis toujours, nous en dépendons à chaque instant, nous en parlons sans arrêt, mais sans avoir jamais cherché [...] à le thématiser, à expliciter son mode de présence, à en analyser les conditions, les effets, la signification, la portée. (2005: 64)

L'ensemble des données que nous venons de présenter sur le petit conte persan nous permet de retracer le parcours narratif et de proposer le schéma canonique de la sanction. Ce dernier est basé sur une sanction anticipée que nous qualifions ici de tensive étant donné son étroite liaison avec la visée et son issue sur une saisie.

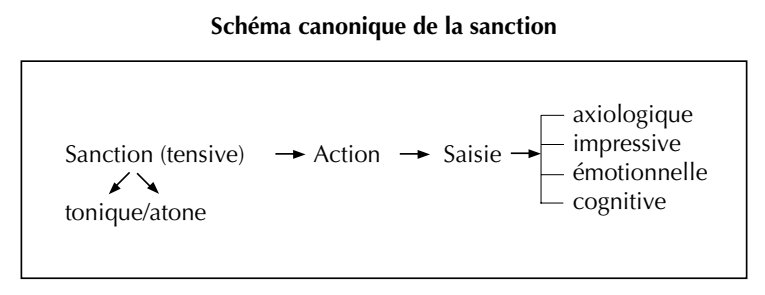

Pour pouvoir terminer cette réflexion, il faut évaluer le rôle de l'anticipation de la sanction dans le bon fonctionnement de l'action. Il s'agit en somme de savoir jusqu'à quel point le contrat doit intégrer une manipulation par sanction anticipée pour que l'action soit réussie. Cette question nous confronte à une différence radicale de la situation: dans le cas de la sanction tensive (tonique) et de la manipulation forcée, on va jusqu'à la réalisation de la punition, alors que dans le cas de l'absence de la sanction tensive (atone), et donc de la rupture de la manipulation, on s'arrête à la virtualisation de la punition. Cette remarque affirme le basculement des modes d'existence selon le type de sanction adopté. Par rapport à la punition réalisée, le sujet ne peut fonctionner que dans une structure embrayée, où il signifie par un programme rétrospectivement établi : aucune attente en perspective. En ce qui concerne la punition virtualisée, le sujet se trouve dans une structure débrayée que l'on peut interpréter comme une situation déformante puisqu'elle renverse tout le contenu narratif et transforme I'axiologie. En effet, avec la version virtuelle de la sanction, on change de registre et un nouveau programme s'ouvre: I'actant collectif essaie d'imaginer le programme du Maître. C'est même celuici qui devient l'objet de l'évaluation du peuple. L'accent est donc mis sur la forme de vie du Maître: "Comment va-t-il agir pour punir le sujet?». Et l'agent est même oublié. De plus, I'anticipation de la sanction invente une sorte de mémoire dont le rôle s'avère essentiel dans l'accompagnement de l'action: mémoire de l'empreinte corporelle.

Dans ce cas, étant donné la «coprésence temporelle ( « tous les jours»), pour emprunter le terme d'U. Eco (1999: 379), et la contiguïté spatiale (le renouvellement de la gifle appliquée constamment au même corps) et actantielle (le rapport corps à corps), nous avons affaire à une entité sémiotique embrayée dont le bon fonctionnement actionnel repose sur la stabilité de la mémoire de l'empreinte corporelle qu'elle produit.

[...] l'empreinte est donc la figure, stable aussi bien dans l'espace que dans le temps, qui permet d'établir le lien entre deux faces, deux statuts, deux états ou deux rôles de la même entité.

(Fontanille, 2004: 165) 
En effet, en ce qui concerne le parcours de la manipulation, nous sommes en présence $d^{\prime}$ 'une force d'unification qui est constitutive d'une «mémoire de l'origine» relevant du "provenir». On peut penser aussi, à la suite de J. Fontanille, que cette mémoire repose sur une tension rétrospective: «Les tensions rétrospectives fixent en quelque sorte le sens de ce qui n'en avait pas encore» (2006: 58).

\section{Conclusion}

Au terme de ce parcours, nous voudrions revenir sur le fait que penser la sanction à la fois comme une manipulation et comme ce qui ouvre le champ de l'action tout en la rendant réalisable par l'intensité de la force somatique revient à se placer au cœur de la problématique tensive et somatique. Ainsi, le renversement de l'ordre des étapes de la narrativité, que nous avons justifié à partir du conte persan, a pour fonction de placer la sanction manipulatoire à l'origine du schéma de l'action. Cela s'oppose à l'aspect mécanique, formel et très logique du parcours narratif standard. En effet, avec les acquis de la sémiotique des passions, de la perception, de la tension, de l'interaction et avec ceux de la phénoménologie ( «la situation de l'homme»), on voit mal aujourd'hui comment les phases de la narrativité et les configurations classiques, telles que nous les connaissons, pourraient rendre compte de tous les aspects de l'objet sémiotique ou des régimes de sens. C'est la raison pour laquelle l'appareil conceptuel de la sémiotique a besoin de s'élargir. D'où la multiplicité des modèles qui correspond à la nécessité de délimiter le champ de la réflexion et de faire reposer la démarche analytique sur une conception «ontologisante».

La présente tentative laisse à penser que la syntaxe narrative, vu le rôle initial et anticipé de la sanction liée à l'activité de I'intensité somatique, relèverait de la problématique tensive et de celle du corps propre. C'est en cela que l'intégration de la grammaire tensive et du corps propre, dans le cadre général de la manipulation et de la programmation, trouve sa justification. C'est ce qui a d'ailleurs donné lieu au schéma canonique de la sanction (page précédente).

En outre, le dédoublement narratif (le programme du Maître et celui du peuple) crée trois spectacles distincts: celui du Maître, celui de la collectivité et celui du lecteur. Chaque scène s'adresse à un destinataire différent: la scène du Maître à l'apprenti, la scène de la collectivité au Maître lui-même et ces deux scènes s'adressent ensemble au lecteur. Il s'agit en fait d'un conte qui contient une leçon pour tout le monde.
Enfin, l'on ne peut pas finir cette réflexion sans évoquer le fait qu' «être persan», c'est être partagé entre deux «formes de vie». La première peut être définie comme une tension-choc contraignante (commencer par la fin) qui anticipe la fin, participe à la réalisation de la sanction, embraie le parcours et fige le sens de la vie dès le début et avant le passage à l'acte tout en empêchant l'emballement imaginaire du sujet de I'action. La deuxième correspond à un relâchement tensif (finir par le commencement) qui s'arrête à la virtualisation de la sanction, débraie le sujet et son parcours, renverse le contenu narratif, s'ouvre sur l'emballement imaginaire de la collectivité et aboutit ainsi à une circulation des scènes. Cela rend le sens de la vie fluide, non figé, dilatant et mis en attente.

\section{NOTES}

1. Les noms les plus importants qui s'inscrivent à l'origine des bouleversements narratifs et qui ont une part importante dans la conception des structures profondes immanentes sont: R. Barthes, A.J. Greimas, C. Bremond, U. Eco, G. Genette, C. Metz et T. Todorov.

2. Il y a aussi un proverbe arabe où la sanction manipulatoire occupe la première place dans le schéma de l'action: «Frappe ta femme tous les matins; même si tu ne sais pas pourquoi, elle, elle le sait très bien » (Nasif, 1996: 53).

3. Nous pensons utile de présenter ici un autre conte persan intitulé «Le Cordonnier et son Apprenti » qui ressemble beaucoup à celui-ci et pour lequel nous avons pu trouver une source écrite.

\section{Le Cordonnier et son Apprenti}

Il y avait un Cordonnier, qui, tous les jours avant de quitter la boutique, punissait son Apprenti en lui rappelant qu'il devait surveiller les cuirs avec attention afin que les chiens ne les volent pas. Un jour, le Cordonnier est parti sans punir son Apprenti. Et ce jour-là, un morceau de cuir a été volé par un chien en raison de négligence de celui qui devait s'occuper de la boutique en l'absence de son Maître. C'est à ce moment-là que l'Apprenti s'est mis à pleurer de peur, s'est réfugié chez les voisins et leur a demandé de s'entremettre auprès de son Maître pour lui. Quand celui-ci a appris la nouvelle, il a appelé son élève au travail et ne s'est même pas fâché contre lui. Très étonnés, les gens du quartier en ont demandé la raison. Le Cordonnier a dit: « les autres jours, je le punissais pour empêcher les chiens de voler les cuirs, mais 
maintenant que le mal est fait, à quoi ça sert de lui en vouloir et de le punir ». (Zolfagari, 2005: 816).

4. La tensivité peut être abordée de deux manières différentes: "Soit comme fondement et généralisation de l'intentionnalité au sens phénoménologique du terme, dont on sait que l'étymon latin in-tendere désigne I'action de "tendre vers", au sens de "viser", approcher quelque chose pour éventuellements'en saisir; $c^{\prime}$ est la structure de tout acte de conscience, qui a son modèle dans la sensori-motricité, les actes moteurs guidés par une visée, pour ne pas dire une "vision", bref un acte de perception ou de saisie anticipée. Soit encore comme généralisation ou eidétisation d'un certain nombre de propriétés physiques permettant de décrire un continuum spatio-temporel: la différence de potentiel dans un flux énergétique donné, la force appliquée à un corps pour en séparer la partie, l'état plus ou moins tendu d'une matière élastique ou extensible, l'état de ce qui menace de rompre, etc. dont le point commun est de conjoindre l'extensité et l'intensité» (Ouellet, 1996: 4).

5. Selon A.J. Greimas et J. Courtés, «la tensivité est la relation que contracte le sème duratif $d^{\prime}$ un procès avec le sème terminatif: ce qui produit l'effet de sens "tension", "progression" [...]. Cette relation aspectuelle surdétermine la configuration aspectuelle et la dynamise en quelque sorte» (1993: 388).

6. J. Fontanille et C. Zilberberg distinguent deux types de valences: les valences subjectales et les valences objectales. Les premières sont celles qui « déterminent les conditions de l'accès à la valeur pour le sujet, ainsi que la valeur de la jonction : de nature essentiellement "rythmique", elles peuvent être identifiées grâce au tempo et à l'aspectualisation de la saisie ou de l'échange » (1998: 23). Les secondes sont celles qui «déterminent dans la morphologie des figures-objets, ce qui les rend propres à accueillir un investissement axiologique, notamment, leur structure méréologique » (ibid.).

7. Selon A. J. Greimas, le déplacement « s'interprète généralement, dans le cadre narratif, comme la manifestation figurative du désir, autrement dit comme la forme narrative de la modalité du vouloir dont se trouve doté le sujet. Dans la mesure où le déplacement a un objet, on peut le définir comme une quête» $(1983: 146)$.

8. Cela va à l'encontre de l'idée de J. Alonso Aldama selon laquelle I'actant collectif relève de l'ordre du discontinu: "cet actant collectif représente l'apparition d'une discontinuité dans le continuum du social; I'articulation que présuppose l'apparition d'un actant collectif équivaut à l'introduction de limites, de démarcations, de différences, dans l'espace "amorphe" et indéfini du social» (2000: 51). D'un côté, l'actant collectif qui nous occupe s'inscrit dans le même continuum tensif dont le déclencheur n'est autre que le Destinateur initial. De l'autre côté, il signifie par rapportà un espace bien défini et déterminéqui est celui d'une sanction pragmatique et d'une tensivité rythmique: une gifle par jour.

9. J.-M. Floch croità « deux grands principes de l'identité: l'idem et l'ipse. L'idem, c'est le même, opposé au variable et au changement; I'idem renvoie en fait à la notion de permanence dans le temps. L'ipse, quant à lui, n'est pas lié à cette idée de permanence dans le temps: il est lié au fondement éthique de soi et à la relation à l'autre» (1995: 40).

10. C. Zilberberg propose d'appeler « introjectif » le temps qui correspond à l'information de l'observateur par le procès et «projectif», celui qui s'interprète comme l'information du procès par l'observateur (1991: 101).

\section{RÉFÉRENCES BIBLIOGRAPHIQUES}

AlONSO AlDAmA, J. [2000]: "Le social instable», Nouveaux actes sémiotiques, no 71-72, Limoges, Presses universitaires de Limoges, 49-64. BERTIN, E. [2003] : « Penser la stratégie dans le champ de la communication. Une approche sémiotique ", Nouveaux actes sémiotiques, no 89-90-91, Limoges, Presses universitaires de Limoges, 15-63.

BRANDT, P.A. [1992]: La Charpente modale du sens, AmsterdamPhiladelphie Aarhus, Benjamins.

BREMOND, C. [1973]: La Logique du récit, Paris, Seuil.

Chalonge, F. de [1996]: "Le corps et la place», dans M. Costantini et I. Darrault-Harris (dir.), Sémiotique, phénoménologie, discours. Du corps présent au sujet énonçant. Hommage à Jean-Claude Coquet, Paris, L'Harmattan, 105-113.

COQUET, J.-C. [1997] : La Quête du sens. Le langage en question, Paris, PUF.

COURTÉS, J. [2003] : La Sémiotique du langage, Paris, Nathan Université. ECO, U. [1999]: Kant et l'Ornithorynque, Paris, Grasset.

FLOCH, J.-M. [1995] : Identité visuelle, Paris, PUF.

Fontanille, J. [1998]: Sémiotique du discours, Limoges, Presses universitaires de Limoges;

[1999] : Sémiotique et littérature, Paris, PUF;

Larose;

[2004] : Soma et Séma, figure du corps, Paris, Maisonneuve \&

[2006] : "Pratiques sémiotiques: immanence et pertinence, efficience et optimisation", Nouveaux actes sémiotiques, n 104-105106, Limoges, Presses universitaires de Limoges, 13-73.

FontANILLE, J. et C. ZILberberG [1998]: Tension et Signification, Liège, Pierre Mardaga.

GenInASCA, J. [1997] : La Parole littéraire, Paris, PUF.

Greimas, A. J. [1983] : Du sens II. Essais sémiotiques, Paris, Seuil.

Greimas, A. J. et J. COURTÉs [1993] : Sémiotique. Dictionnaire raisonné de la théorie du langage, Paris, Hachette.

LANDOWSKI, E. [2005]: "Les interactions risquées», Nouveaux actes sémiotiques, $\mathrm{n}^{\mathrm{O}}$ 101-102-103, Limoges, Presses universitaires de Limoges, 7-100.

MONTESQUIEU [(1721) 1992] : Lettres persanes, Paris, Flammarion.

NASIF, E. [1996]: Les plus beaux discours qui existent sur les femmes, Beyrouth, Dar Al-djil.

OUELLET, P. [1996] : « Pour une sémiotique tensive, les gradients du sens», Nouveaux actes sémiotiques, no 46-47, Limoges, Presses universitaires de Limoges, 3-12.

PARRET, H. [2001]: «Présence», Nouveaux actes sémiotiques, no 76-7778, Limoges, Presses universitaires de Limoges, 9-126.

Propp, V. [(1928) 1970] : Morphologie du conte, Paris, Seuil. ZILBERBERG, C. [1991]: «Aspectualisation et dynamique discursives», dans J. Fontanille (dir.), Le Discours aspectualisé. Actes du colloque "Linguistique et sémiotique I» tenu à I'Université de Limoges (1989), Amsterdam et Philadelphie, Presses universitaires de Limoges et Benjamins, 83-104;

[2006]: "Retour sur Bonne pensée du matin de Rimbaud», Nouveaux actes sémiotiques, $n^{\circ} 107-108$, Limoges, Presses universitaires de Limoges, 25-72.

Zolfagari, H. [2005] : Les Contes populaires, Téhéran, Mazeyar. 